
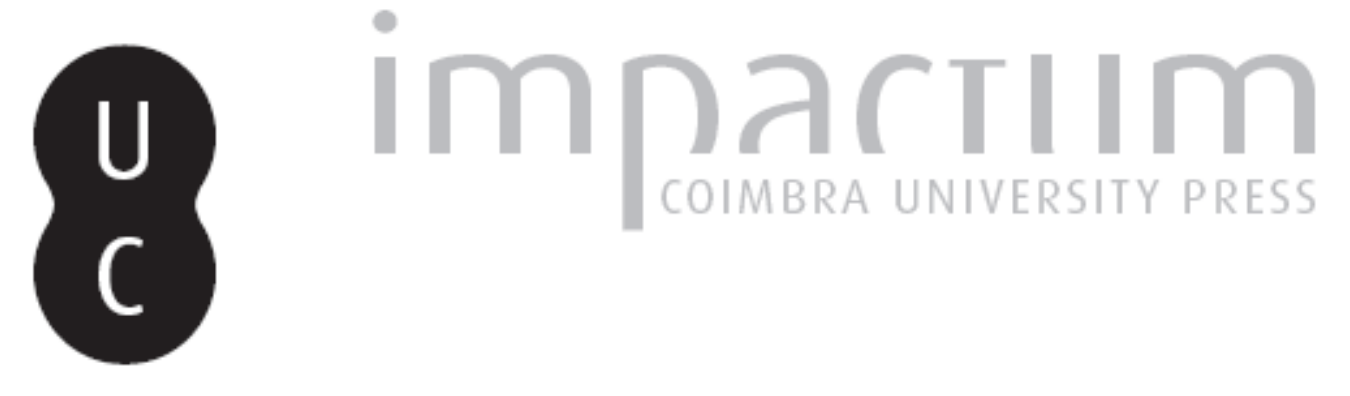

\title{
Riscos de fumos de incêndio: actualidade e controvérsias nas intoxicações $\begin{array}{ll}\text { Autor(es): } & \text { Bandeira, Romero; Leão, Rui Ponce; Gandra, Sara; Reis, Ana Mafalda; } \\ \text { Gandra, Romero }\end{array}$
}

Publicado por: Associação Portuguesa de Riscos, Prevenção e Segurança

URL persistente:

URI:http://hdl.handle.net/10316.2/36164

DOI:

DOI:http://dx.doi.org/10.14195/1647-7723_16_16

Accessed : $\quad$ 26-Apr-2023 14:00:18

A navegação consulta e descarregamento dos títulos inseridos nas Bibliotecas Digitais UC Digitalis, UC Pombalina e UC Impactum, pressupõem a aceitação plena e sem reservas dos Termos e Condições de Uso destas Bibliotecas Digitais, disponíveis em https://digitalis.uc.pt/pt-pt/termos.

Conforme exposto nos referidos Termos e Condições de Uso, o descarregamento de títulos de acesso restrito requer uma licença válida de autorização devendo o utilizador aceder ao(s) documento(s) a partir de um endereço de IP da instituição detentora da supramencionada licença.

Ao utilizador é apenas permitido o descarregamento para uso pessoal, pelo que o emprego do(s) título(s) descarregado(s) para outro fim, designadamente comercial, carece de autorização do respetivo autor ou editor da obra.

Na medida em que todas as obras da UC Digitalis se encontram protegidas pelo Código do Direito de Autor e Direitos Conexos e demais legislação aplicável, toda a cópia, parcial ou total, deste documento, nos casos em que é legalmente admitida, deverá conter ou fazer-se acompanhar por este aviso.

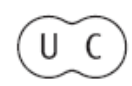




\section{territorium}

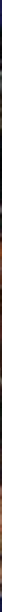

16

Revista da Associação Portuguesa de Riscos, Prevenção e Segurança 2009 


\section{RISCOS DE FUMOS DE INCÊNDIO. ACTUALIDADE E CONTROVÉRSIAS NAS INTOXICAÇÕES*}

Romero Bandeira

Medicina de Catástrofe - ICBAS/CPorto; Presidente do Conselho Científico e Pedagógico - ENB lmedcat@idbas.up.pt

Rui Ponce Leão Mestre em. Saúde Capacional; Docente - ESS Gaia/Instituto Piaget

Sara Gandra

Mestre emMedicina de Catástrofe pelo ICBAS/UP; Enfermeira do Serviço de Ciidados Intensivos I - HGSA/

GHorto

Ana Mafalda Reis

Dautoranda, Medicina de Catástrofe - ICBAS/CPorto; Neurorradiologista - HPH/trSMatosinhos

Romero Gandra

Jirista - Inspector Estagiário PJ; Formador ENB

\section{RESUMO}

As vítimas de um incêndio manifestam comumente lesões por inalação de fumos, sendo esta a causa mais frequente de morte, quer em pacientes que apresentam queimaduras, quer na ausência das mesmas.

A inalaçãa de fumos pode ocasionar lesão epitel ial directa na mucosa respiratória comperda dos cílios epitel iais brônquicos e diminuição db factor tensicactivo alveolar. Prodzen-semicroatelectasias e por vezes atelectasias que se complicam por edema mucoso, assim como, dostrução mecânica pelo tecido descamado e respectivas secreçães, originandb amulativamente ma insuficiência respiratória agudamultifactorial (AnA, 2008) .

As principais controvérsias tên-se centradb no tratamento das intoxicações por co e HoN que, como se comprovar nas últimas décadas, se encontram conbinados na maior parte dos incêndios commateriais conbustíveis sintéticos (Drou, 2008) .

Abordaremos as intoxicaçães por co e HON, que pela sua maior frequência e morbi-mortalidade consideramos seremmais relevantes.

Palavras chave: CO, controvérsias, HON, intoxicações, riscos

\section{RÉSUMÉ}

Les victimes d'un incendie manifestent comunément des lésions dues aux inhalations de fumées, celles-ci étant la cause la plus fréquente de mort dhez les patients brûlés au non.

L' inhalation de fumés peit provoquer des lésions épithéliales directes sur la muquase respiratoire avec perte des cils épithéliales brondhiques et une diminution du facteur tensioactif alvéolaire. Des micro-atélectasies et parfois de atélectasies sont produits. Ceux-ci peuvent s'aggraver avec l'oedème muqueux, au par dostruction mécanique provoquée par le tissu desquanéet leurs sécrétions, provocant ainsi une insuffisance respiratoire aiguëmultifactorielle $\left(\mathrm{AmA}_{\mathrm{MA}}, 2008\right)$.

Ies controverses principales sont centrées sur le traitement des intoxications par co et HCN, qui, conme vérifiéau caurs des demières amées, se retrouvent savent associés lors des incendies comportant des matériaux conbustibles synthétiques (Drou, 2008) .

Nous aborderons les questions d' intoxications par co et Hav car nous les considérons très importantes vu leur fréquenceet leurmorbi-nortalité.

Mbts-clé: CO, controverses, HCN, intoxications, risques

\footnotetext{
* Commicação apresentada ao V Encontro Nacional e I Congresso Intemacional de Riscos.
} 


\section{ABSTRACT}

The victims of a fire comonly reveal injuries for inhalation of smoke which is a frequent cause of death in patients with or withat laming problems.

The inhalation of smoke can cause epithel ial injuries in the respiratory mucosa with loss of brondhial epithelial cilia and reduction of the alveolar tensicactiv factor. Microatelectasis and atelectasis can be complicated because of the mucous edena, as well as, the blodkage medhanics for the production of desquamated tissue and respective secretions,

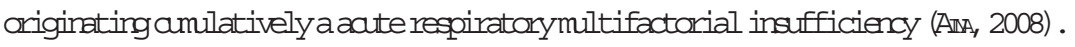

The main controversies have been focused on poisonings treatment with $\mathrm{CO}$ and HON that are combined with synthetic conturstible materials, as it has been proved in the last few decades (Dru, 2008) .

We will approach the poisonings for $\mathrm{CO}$ and HCN, that for its bigger frequency and morbi-mortal ity we consider tobemore relevant.

Keywords: CO, controversies, HCN, poisonings, risks

\section{Introdução}

O combate a Fogos é uma intervenção frequente dos Banbeiros, quer sejam Profissionais au Voluntários, envolvendb, porvezes, centenas de indivíduos.

Inalação de fumo num Incêndio:

- Ambrtalidade, principalmente ligada aos fumos mais do que ao calor a aos riscos traumáticos

- História Clínica: em Providence, 23 de Març̧o de 2006, no Estado de Rhodes Islands (USA), os Bombeiros intervêm em 3 fogos relativamente clássicos: um restaurante, um fogo num contentor de lixo e um fogo numa casa.

- Na sequência da primeira intervenção um Bombeiro apresentou perturbações do comportamento com um discurso incoerente. conduzidb ao Serviço de Urgência fez teste para cianetos que se apresentau positivo comuma taxa tóxica; no momento do $2^{\circ}$ incêndio vários Bonbeiros tiveram sinais clínicos tipo fadiga, cefaleias, vertigens, indisposição, transpiração, rubor facial... no $3^{\circ}$ incêndio, um Bombeiro apresentou uma paragem cardio-respiratória, associada a atros sinais clínicos.

- Todbs apresentaram una taxa elevada de cianeto no sangue.

In: Lévy F (2008)

Entexto anterior (Banwira et al, 2007) saliental-se o Risco de Intoxicação pelo Monóxido de Carbono. contudb este não é o único aspecto relevante de entre os diversos agentes tóxicas a que o Bombeiro pode estar exposto numa intervenção em Fogos, sejam estes unbanos, industriais, nurais, florestaisaratros.

A detecção do co pode hoje ser real izada no terreno contoda a facilidide (fig. 1, 2, 3)

A conlustão pode, tanbém, prodzirir cianetos tal como foi exposto en Comunicação apresentada no nosso V Fncontro Nacional e I congresso Intemacional de Riscos 2009.

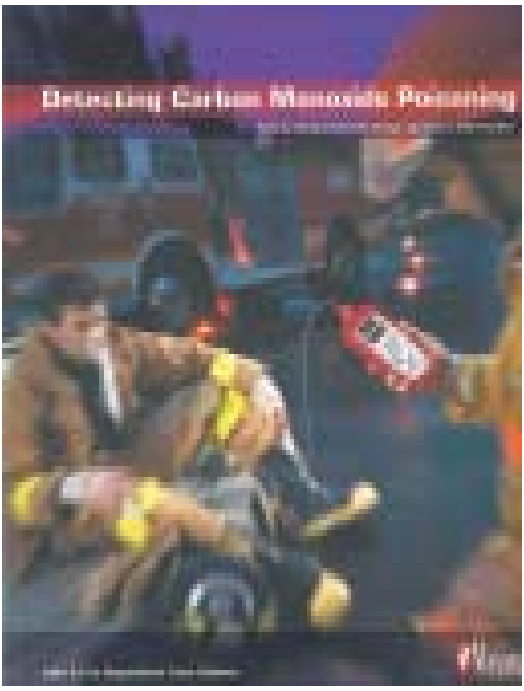

Fig. 1

\section{Intoxicações por CO e HCN}

De acordo com Sebastián Miño, da Unidad de Toxicologia Clínica do Hospital Clínico Lniversitário de Zaragoza em Comunicação subordinada ao título "Composición Química de los Gases y Mecanismos Fisiopatológicas de Acción" apresentada nas XVIII Jomadas Nacionals de Sanitários de Bomberos, integradas no "250 Aniversario da Asistencia Médica del Servicio contra Incendios de Salvamento y Protección Civil" realizadas en Novembro de 2008 em Zaragoza, podemos considerar:

1- Toxicidade dos gases segundo o seu mecanismo de acção:

- Combustíveis (latano, propano, ...) - deslocam $\mathrm{O}_{2}$ do ar ambiente.

- Tóxicos (CO, HCN, ...) - absorvem-se para o sangue exercendb o sev efeito a nível sistémico, 
bloqueando o transporte e o aproveitamento do $\mathrm{O}_{2}$.

- Imitantes (cloro, formaldeídb, amoníaco, ...) exercem uma acção irritante sobre o trato respiratório e sobre todas as mucosas com as quais entram em contacto.

2- Componentes do Fogo: Chamas, gases luminosos, calore fumo.

3- Quanto ao Fumo: Mistura de gases, vapores, pequenas partículas líquidas e sólidas em suspensão.

4 Quanto aos Gases Irritantes que se produzemnum Incênoio: Áciob Clorídrico, Fomaldeído, Acroleína, Isocianatos, $\mathrm{NO}_{2}, \mathrm{HF}, \mathrm{COCl}_{2}$ (Fosgéneo).

Estes provocam desde irritação das Vias Aéreas Superiores, a edema, broncospasmo, podendo mesmo levar a lesão alveolar difusa desencadeando um ARDS.

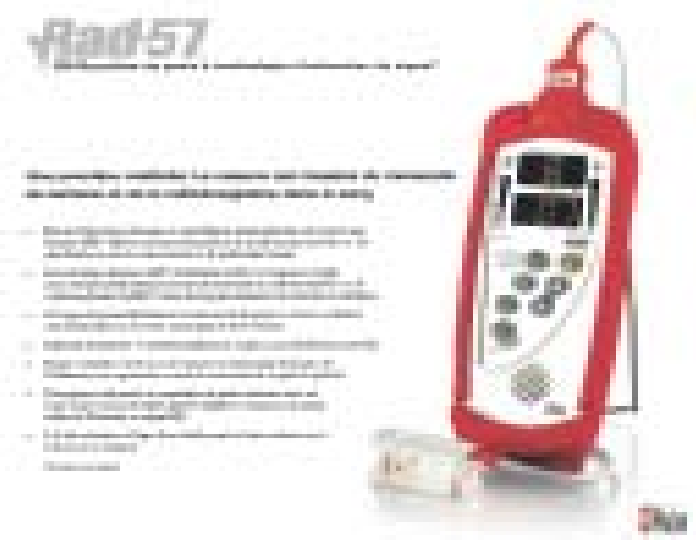

Fig. 2
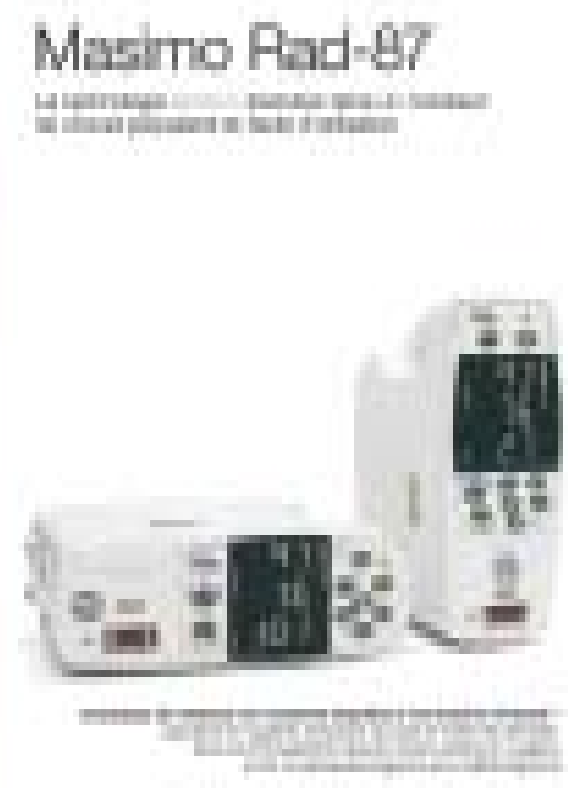

Fig. 3
5 - Fisicpatologia (CO)

- Uma vez no Sangue o co une-se à Hemoglobina com uma afinidade de 210-270 x superior à do $\mathrm{O}_{2}$ formando um composto denominado carboxihemoglobina (COHb) .

- Desta maneira o $\mathrm{O}_{2}$ disponível não se pode unir à Hemoglobina e o seu transporte aos tecidos está impossibilitadb.

- Pode ainda ligar-se a autras hemoproteínas (mioglobina, citocromo-axidase, citocromo P450 e hidroperoxidase) em 15\% a 20\%.

Conformenos transmitiuArtigas Ama, daAssistência Médica do Serviço contra Incêndios, Salvamento y Protección Civil del Ayuntamiento de Zaragoza em comnicação intitulada "Intoxicatión por Humos" nas já aludidas Jomadas de 2008, em Zaragoza, o tratamento para uma intoxicação pelo co deve ser o seguinte:

- Retirar opaciente da fontede CO, suportevital e administração de $\mathrm{O}_{2}$ a 100\%, sem esperar confirmação toxicológica.

- Duração mínima 6 h.

- A semi-vida da COHb no ar ambiente é de 320min., diminui par os 75min com O2 a 100\% e em $\mathrm{O}_{2}$ hiperbárico cai para 20 a $25 \mathrm{~min}$.

- Via periférica com soro glicasadb a 5\%.

- Mbnitorizar Sinais Vitais + ECG.

- Intubação oro-traqueal: ARDS, Shock persistente, cona, convilsões, edenapulmonar.

Quanto ao tratamento da intoxicação pelo HaN devem ser relevadbs os princípios seguintes:

- O a 100\% por máscara com reservatório.

- Retirar a roupa e lavar a pele comágua.

- En caso de convulsões - BDD.

- Antílotos:

- Agentes meta-hemoglobinizantes

- Tiossulfato de sódio

- Hidroxicabalamina

o Cianeto de Hidrogénio foi isolado pela primeira vez em 1782, tendo o investigador falecido em 1786 por envenenamento por Cianeto. Nbipoleão III terá sido o primeiro a uti lizá-lo cono arma de guenra; mais tarde foi utilizado na $1^{\text {a }}$ Guerra Mundial. O govemo Nazi utilizou-o como agente de genocídio com o nome de Zzkklon B. A uti i ização terapêtica de Nitropnussiato como vasodilatador nas crises hipertensivas pode originar intoxicação: 5 moléculas de Cianeto por molécula de nitropnussiato (Hrwnv et al, 2007) .

Os Cianetos ( (N-) são extremamente tóxicos por ligação ao ferro da hemoglobina, impedindo assim a captação do oxigénio.

Emutilização industrial pode ser utilizado para a extracção de arro (arro + cianeto de potássio) formandb $|\mathrm{Au}(\mathrm{aN}) 2|$-. Édiferentedos Cianatos auFulminatos, derivadbs do ácido Ciânico ou Fulmínico (HOON) , um explosivo instável (OxfordDictionary of Science, $5^{\mathrm{a}}$ ed, , 2005) . 
A toxicidade de uma dose depende da forma de Cianeto (gás ou sal) , da via de administração e do tempo de exposição. Para um adulto a dbse oral letal de KCN (Cianeto de Potássio) é de 200ng e a dose letal inalada de 270ppm ( $\mu \mathrm{g} / \mathrm{mL}$ ) pode resultar em morte imediata (HoFmpan et al, 2007) .

Em Portugal a Norma Portuguesa NP 1796 de 2007 - Segurança e Saúde no Trabalho - Valores Limite de Exposição Profissional a Agentes Químicos, $4^{a}$ edição - Setenbro de 2007 - Instituto Português da Qualidade, com o Termo de Homologação No . 332/2007, de 200709-26, indica na página 9, como Substância "Ácido cianídrico e sais de cianeto, expressos en $\mathrm{NN}^{\prime}$ as valores VIE (Valor Limite de Exposição) de CM (Concentração Máxima) de 4, 7ppom para o Ácido Cianídrico e de CM 5mg/m3 para os Sais de Cianeto. Não é indicado o VLE-MP (Média Ponderada) nem o VLE-CD (VLE de Curta Duração) . Como base para a definição do VLE indicase "Irritação do TRS; cefaleias, náuseas e efeitos na tiróidel" (IRS-TractoRespiratório superior) .

As enzimas tiossulfato-ciananeto sulfurtransferase e $\beta$-mercaptopinuvato-cianeto sulfintransferase levam à formação de tiocianato que pode ser excretado porvia renal.

Curiosamente, tal como o Monóxido de Carbono, também pode resultar de produção endógena (HoFrnqN etal 2007).

Já em 2002 no Manual de Trauma do Instituto Nacional de Energência Médica, referia-se na página 45: "Ventilação

A principipal causa de morte num incêndio éa inalação de gases nocivos e não a lesão térmica em si.

Apesar de assegurada a permeabi 7 i dade da via aérea, a venti laçãopock não ser eficaz đeviob à inalação de fumo. O manáxidb de carbono e o cianeto de hidrogénio (libertadb na conbustão de polímeros sintéticas - por ex: revestimentos de cozinha, interiores de autamóvel) são as princippais produtos tóxicos implicadbs. "... "A intoxicação por cianetos também reduz a disponibi 7 idade de $\mathrm{O}_{2}$ e constitui autra indicação para oxigenoterapia."

As preocupações existentes sobre as actividades dos Bombeiros são também patentes num trabalho de J. LATrnev et al (2009), de cujo resumo extractámas It is well known that fire fighters are potentially exposed to variaus carcinogenic agents at a fire scene. An almost unheedbd issue, however, is fire fighters' exposure to carcinogenic agents in smoke diving simulators. Biomonitoring (urinarymuconic acid, 1-naphthol and 1-pyrenol), dermal (polycyclic arcomatichydrocarbons) and occupational hygiene measurements (cyanides, hyckrogen cyanide, polycyclic arcamatic hychrocarbons, volat ile organic compounds and formaldehyde) were used to determine how the lamingmaterial, the type of simulator andprotective clothing used affect fire fighting trainers' exposure.
Trata-se, pois, de um trabalho em que é focada a exposição a tóxicos em treino de Bombeiros em 'Mergulho' num simulador de Fogo e respectivos agentes tóxicos, incluindb carcinogénios commonitorização de alguns parâmetros.

Igualmente é referida a relevância das Intoxicações por Cianetos, quer estes resultem da combustão de algunsmateriais, quer surjamemresultadb deacidentes, acidentes industriais, acçõesmi i itares e tenroristas; foi, também, utilizado em campos de concentração (SzNIIACO, 2003 a, b) . Também nestes casos pode ser solicitada a intervenção de Bonbeiros.

Imediatamente após a inalação de Cianeto de Hidrogénio ou após alguns minutos ou mesmo horas após a ingestão de sais de cianeto auplantas au químicos cianogénicos surgem os sintomas: cefaleias, náuseas, vómitos, ansiedade, confusão e colapso. Inicialmente pose surgir hipertensão e taquicardia que progridem para hipotensão, bradicardia e apneia. O dbente pode apresentar Hịpervent i lação por estimulação do Sistema Nervoso Central. O hálito a "amêndbas amargas" pode surgir tal como o mesmo odor no vómito, mas não é fiável as Cianetos causam hipoxia celular actuando ao nível da cadeia respiratória mitoconodrial, bloqueando a citocramo-oxidase. A manutenção da permeabil idade das vias aéreas e da qualidade e profundidade da respiração. Amonitorização das concentraçães arteriais de dióxido de carbono (CO2) e de oxigénio (O2) , o pH arterial, a oiurese, amonitorização electrocarotiográfica (ECG) , a colocação de cateter venoso, a collheita de sangue venoso para determinação da glicemia, ionograma, creatinina sérica, função hẹática e da eventual, seloem que demorada, quantificação do cianeto são medidas gerais a efectuar (Mrran, 2008 e Oson, 2009) .

contubbara além de inibir a citocrono-oxidase inibe, ainda, a desidrogenase do ácidb succínico, a superóxidb dismtase, a anidrase carbónica.

A intoxicação por Cianeto, tal como pelo Mbnóxido de Carbono, pode levar ao aparecimento Parkinsonismo secundário (Da Log Mhon et al., 2008) .

O tratamento das intoxicações por Cianetos foi, durantemito tempo, umprdblena difícil de resolver. De acordo com Osson (2009) para além da administração de Oxigénio (O2) a 100\%, estão disponíveis o conjunto Nitrito deAmilo, 0,3ml para inalação + Nitrito de Sóciio $300 \mathrm{mg}$ (IV) + tiossulfato de sódio 12,5g (IV) e a Hidroxocobalamina (Cyandrit), aprovada pela FDA nos Estados Unidos em Dezembro de 2006.

É conhecida (HoFmMan, 2007) a utilização do Oxigénio Hịperbárico nas intoxicações combinadas de Mbnáxido de Carbono e Cianeto, passível por exemplo em casos de inalação de fumos. Com a uti lização de Oxigénio Hịperbárico combinado com nit rato de sódio + tiossulfato de sódio resultau, de acordb com o autor citadb, em sobrevida sem sequelas neurológicas. 
A acção quelante do cobalto sobre o cianeto é conhecida desde 1894 (Hornvan, 2007) . O cobalto é um dos átomos da Hidroxocobalamina, a Vitamina B12a (aquacobalamina, $\mathrm{Cbl}$ (III) H2O, vitamina B12a), precursor da Vitamina B12. A Vitamina B12 foi isolada em pelo cientista dos USA Karl Forres em 1948, sob a forma de Cianocobalamina.

De acordo com SHFPHFD e Vewrz (2008), Cyanide poisoning can rapidly cause death. Heving an effective atiobte readily available is essantial for facilities that provide emergency care. In cases of cyanide ingestion, both the nitrite/thiosulfate conbination and hydroxocobalamin are effective antiobtes. Hydroxocobalamin offers an improved safety profille for chil dren andpregnant women. Hydroxocobalamin also appears to have a better safetyprofile in the setting of cyanidepoisoning in conjunction with snoke inhalation. However, ament data are insufficient to recamend the empiric adninistration of hydroxocdbalamin to all victims of smoke inhalation.

\section{Hidroxocobalamina (Cyanokit)}

Nos úl timos anos surgiu uma nova hipótese terapêutica a Hidroxocdbalamina, uma das formas de Vitamina B12 disponíveis no mercadb, mas em dose elevada e por via endbvenasa ("Cyandkit") . En França o preço ao público é de 671, 75 Firros por 2,5ml. A hidroxocdbalamina permuta de modo inreversível um ião hidroxi por um ião cianeto, formandb cianocdbalamina atóxica.

Aprovado pela European Medicines Agency, o Relatório Público Erropeu de Avaliação para o Cyandkit (figs. 4 e5) encontra-sedisponível paraopiblico.

contudo, a relevância do problema justifica a transcrição de partes do documento: "... Cyanokit é umpó para solução para perfusão (administração gota a gota numa veia). Contém a substância activa hidroxocobalamina (vitamina B12a)...".

\section{Para quéétilizadb o Cyanokit?}

o cyanokit é ut i lizado camo antídbto para tratar intoxicação conhecida ou suspeita por cianeto, uma substância química extremamente tóxica.

A intoxicação por cianeto resulta, habitualmente, da exposição a fumo de incêndio, da inspirração au da ingestão de cianeto, au do seu contacto com a pele au com as membranas mucosas (as superfícies húmidas do corpo, tais como o revestimento da bocal) .

o medicamento só pode ser obtido mediante receitamédica.

\section{Camo se utiliza o Cyanokit?}

o Cyanokit é administrado como tratamento de emergência, logo que possível após a intoxicação. É
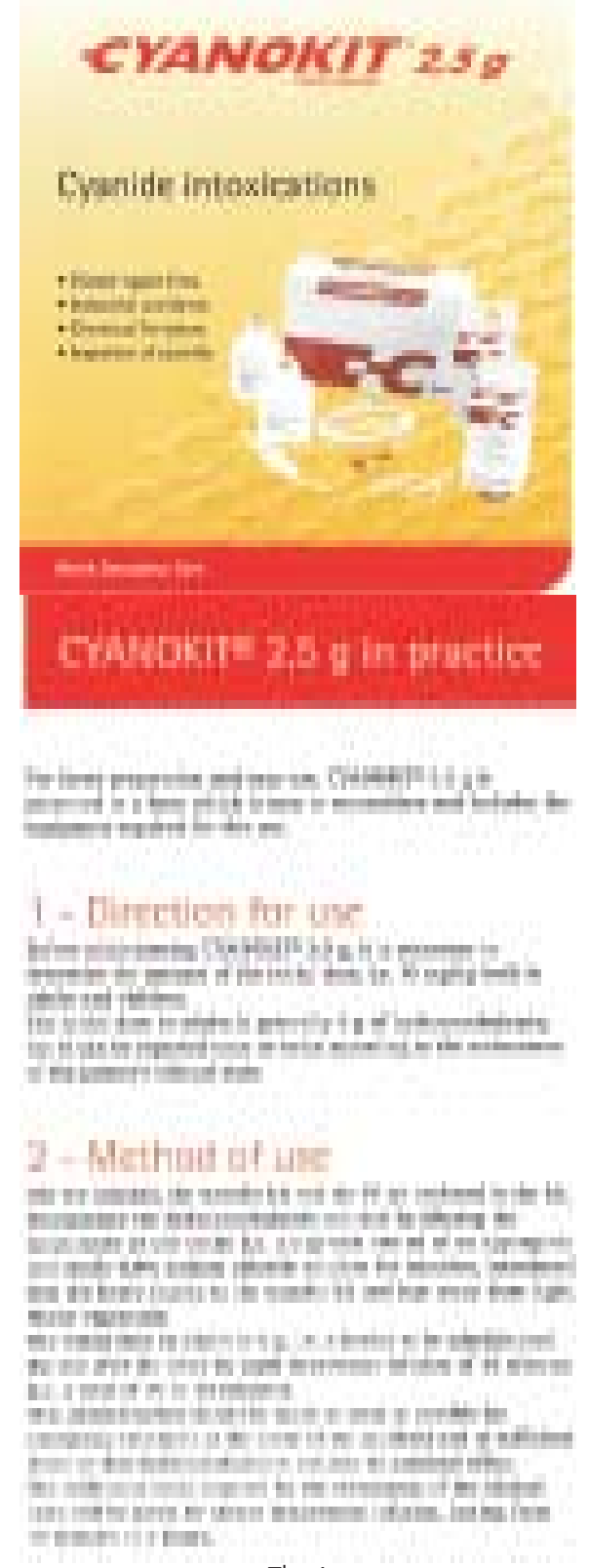

Fig. 4

administrado como perfusão intravenosa durante 15 mintos. Enadultos, a dbse inicial édé 5g. Emcrianças, a dose inicial é de $70 \mathrm{mg}$ por quilograma de peso corporal, até à dose máxima de $5 \mathrm{~g}$. Poderá ser administrada uma segunda dose, dependendo da gravidade da intoxicação e da resposta do dbente.

A segunda dose é administrada durante imperícob de entre 15 minutos e duas horas, dependendo da condição do dbente. A dase méxima é de log em adul tos e de $140 \mathrm{mg} / \mathrm{kg}$ em crianças até um máximo de $10 \mathrm{~g}$.

o Cyanokit é administrado em conjunto com medidas apropriadas para descontaminar e suportar o dbente, incluinob o fomecimento de oxigénio para o obente respirar. 


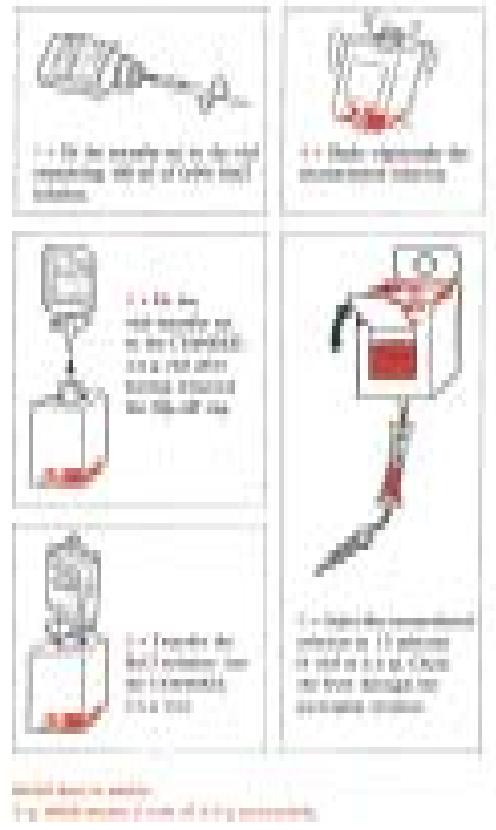

Fig. 5

\section{Camo funciona o Cyandkit?}

A substância activa do Cyanokit, a hidroxocobalamina, reage com o cianeto no organismo. Esta reacção forma cianocobalamina, um composto não tóxico que é removido do organismo através da urina. Isto baixa os níveis de cianeto no organismo e impede que o cianeto se fixe à enzima das células denominada citocramo oxidase, a qual é importante para proporcionar energia às células. Isto ajuda a reduzir os efeitos da intoxicação por cianeto. A hidroxocobalamina (vitamina B12a) tem sido utilizada como suplemento vitaminado desde a década de 50. ... Num estudo de 69 doentes, a intoxicação por cianeto deveu-se à exposição ao fumo de um incêndio. Neste estudo, a condição dos doentes no local do acidente foi comparada com a condição após a perfusão com Cyanokit e curante as três dias subsequentes.

Foram estudados mais 14 dbentes a ja intoxicação por cianeto se deveu a outras causas que não a inalação de fumo; a maioria destes doentes tinha tomado cianeto durante uma tentativa de suicídio. A informação sobre estes dbentes foi dbtida a partir dbs seus registos médicos, tal camo introaurzidos em bases de dados de dbis haspitais franceses.

\section{Qual o benefício demonstrado pelo cyanokit durante} as estudas?

No estudo da inalação de fumo, a resposta ao cyanokit foi avaliada camo "positiva" em 31 dbentes (45\%), "parcial" em 15 (22\%) e "ausente" em 10
(15\%). A respoasta foi desconhecida nos restantes 13 doentes. Cinquenta dos doentes deste estudo sobreviveram. A sobrevivência foi mais provável nos doentes que receberam Cyanokit antes da paragem cardíaca, que apresentavam sintomas menos graves de lesão cerebral e que tinhamníveis mais reduzidos de cianeto no sangue. Dois doentes sobreviveram apesar de terem sofrido uma paragem cardíaca antes de receber Cyanokit. Os sintamas de lesão cerebral foram resolvidos em 38 dos 66 dbentes.

Dos 14 doentes cuja exposição ao cianeto se deveu a outras causas que não a inalação de fumo, 10 sobreviveram, incluindo sete dbentes comníveis "letais" (potencialmente fatais) de cianetono sangue."

Porém deve ser relevado de acordo com Mbran (2008) , que em casos fatais as níveis sanguíneas de Cianeto são superiores a 1-2mg/mL.

"Os quatro doentes que morreram apresentavam níveis elevados de cianeto no sangue e os seus corações tinham parado ou apresentavam paragem respiratória antes de terem recebido cyandkit.

\section{Qual é o risco associado ao Cyanokit?}

Devido à cor vermelha intensa da hidroxocobalamina, a maioria dos doentes apresentará uma descoloração vermelha-escura da pele e das membranas mucasas durante até 15 dias, bem como da urina durante até 35 dias, após a administração de Cyanokit. ..."

Foi autorizada a sua uti l ização na Europa desde 23 de Novembro de 2007.

Através do Infarmed (http://www.infarmed.pt/ formlario) épossível verificar as características do "CYANOKLT" autorizado em Portugal: Pó para solução para perfusão $2,5 \mathrm{~g}-$ I.V.

Contudo, nem todos os autores referem esta hipótese terapêutica (Hrst, 2008) .

\section{Sintese}

Em resumo e de acordo com Augustine e WALSH (2006) , apresentamos os dois seguintes quadros elucidativos:

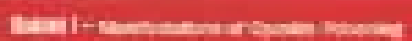

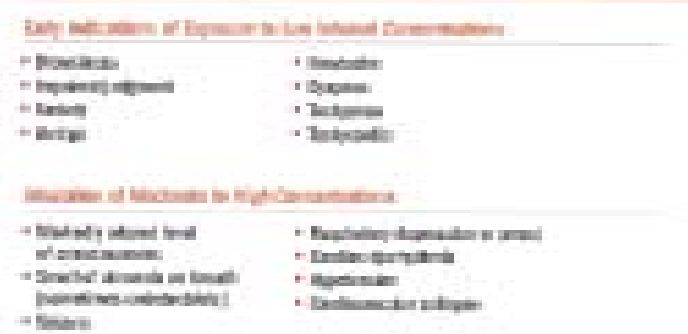




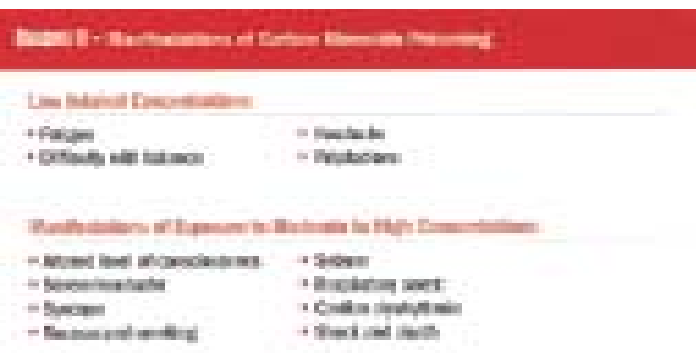

Bem como, de acordo com Forrtw et al. (2006), relevamos:

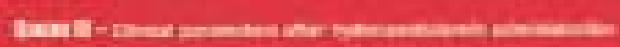

\begin{tabular}{|c|c|c|c|c|}
\hline & 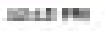 & nere & 표 & 40 \\
\hline miniln & 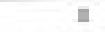 & 1 & 1 & 1 \\
\hline the & III & III & 4 & II \\
\hline 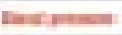 & IME & $114=$ & arse & Lyz \\
\hline tal & H1: & $m$ & $\mathrm{mth}$ & $\mathrm{m}$ \\
\hline tan & a & & 4 & \\
\hline
\end{tabular}

Em complemento da sistematização feita podem ser consultados as Qurres IV, V e VI (Bad, 2008) :

\section{Quabab IV}

Liste académique des signes et symptỏmes induits par les agents asphyviants.

\begin{tabular}{|c|c|c|c|c|c|}
\hline & Hypoxie & $\mathrm{CO}_{2}$ & co & $\mathrm{HCN}$ & Methl \\
\hline Celphaleses & + & + & + & + & + \\
\hline $\begin{array}{l}\text { Troables gistno- } \\
\text { intestinaux }\end{array}$ & & & + & + & + \\
\hline Vertiges & + & + & + & + & + \\
\hline Confusion & + & + & + & + & $\bullet$ \\
\hline Syncope & + & + & + & + & + \\
\hline Convulsions & + & + & + & + & + \\
\hline Coma & + & + & + & + & 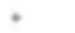 \\
\hline Hypoension & + & + & + & + & + \\
\hline Mrythmie cardiague & + & + & + & + & + \\
\hline Ischemie myocandigue & + & & + & + & + \\
\hline Polypnée & + & & + & + & + \\
\hline
\end{tabular}

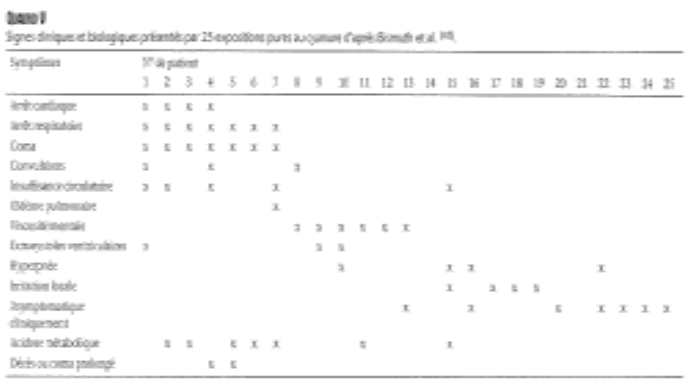

Quecas UI

Valeur diagnostique de la présence de suies vis-à-vis d'une intovication oxycarbonée et d'une intovication cyanínydrique. D'après Baud et al. [so].

\begin{tabular}{|c|c|c|c|c|}
\hline & $\begin{array}{l}\text { Senısibilite } \\
\text { (\%) }\end{array}$ & $\begin{array}{l}\text { Spécificitité } \\
\text { (अ) }\end{array}$ & $\begin{array}{l}\text { Yaleur } \\
\text { prédictive } \\
\text { pesitive [os] }\end{array}$ & $\begin{array}{l}\text { Valeur } \\
\text { précíctive } \\
\text { aégative } \\
\text { (\%) }\end{array}$ \\
\hline $\begin{array}{l}\text { latosication } \\
\text { oxycartwoné }\end{array}$ & 33 & 63 & 43 & 92 \\
\hline $\begin{array}{l}\text { Intousication } \\
\text { cyanlingdrique }\end{array}$ & 98 & 56 & 28 & 99 \\
\hline
\end{tabular}

\section{Controvérsias}

Extractamos da Comunicação da Dra Ana Ferrer Dufol, da Unidad de Toxicologia Clínica do Hospital Clínico Universitário de Zaragoza intitulada, "Controvérsias en el tratamiento específico de la inhalación de Humo" e igualmente apresentada nas já aludidas Jornadas de Novembro de 2008, o seguinte, quanto ao tratamento da intoxicação por Monóxido de Carbono.

Os Protocolos apresentam duas alternativas de Oxigenoterapia:

- Normobárica, máscara com reservatório ou intubação a 100\% (3h) ;

- Hiperbárica 3atm (1h) - Glasgow 7 ou < considerar igualmente a existência de antecedentes cardiovasarlares.

No concemente ao tratamento da intoxicação por Cianetos evidencia-se que:

Dos três tipos de antídbtos propostos referidos já no final do Sec. XIX, tem-se vindb a disautir vantagens e inconvenientes.

- Oxidantes: inconvenientes do emprego de nitritos (sódio e amilo) com seus efeitos secundários

- Sulfuretos (tiossulfato sódico) - tratamento complementar; efeitos adversos: hipotensão ligeira, náuseas e vómitos

- Quelantes de cobalto (hidroxicobalamina)

o ácido cianídrico é, ainda, um risco desconhecido para o Bombeiro em intervenção. Toda a problemática inerente ao mesmo só tem vindo a ser estudada em extensão e profundidade nos últimos anos, sobretudo em países possuem serviços de Medicina Oaupacional bem desenvolvidos nesta área.

Nesta ordem de ideias e referindo os autores franceses Migarbane, FortIn e Hacheiaf (2008) quanto aos exames complementares, evidenciamos:

- Acidose metabólica +++

- Aumento dos lactatos sanguíneos:

- >8mol/1 (intoxicação por sais de cianeto) e >10mol/1 (inalação de fumos de incêndio) .

- Doseamento dos cianetos sanguíneos: limiar tóxico (40mol/1) e limiar letal (100mol/1) .

- Pensar em ter disponíveis, se passível, 2 tubos para doseamento ulterior de cianeto e lactatos sanguíneas.

Os Efeitos Combinados do CN e do CO são importantes (Penney 2009) .

o aN e o co inibem a geração da energia celular, embora por mecanismos diferentes (o av dentro de células e o co principalmente no sangue) ; não seria esperado que uma importante interacção aditiva ou sinérgica pudesse ocorrer. Isto porque qualquer dos tóxicos possui uma cadência de velocidade específica 
de actuação. Todavia, as estudbs em animais mostram algum grau de adição. Em primatas o tempo para incapacitação por HON está reduzido pela presença de altas concentraçães de co. Un dos motivos é que a hiperventilação induzida pelo đN aumenta a taxa de captação quer do đN quer do Co. Isto provavelmente também acontece porque o traçado dos efeitos da absorção para o aN é totalmente diferente do do co quando este actua sozinho, o qual não causa hiperventilação (quer taquipneia ou hipenpneia) . Com baixas concentrações de đN (< 100 ppm) o tempo para incapacitação é > 20 minutos, mas com elevadas concentrações de QN (por exemplo 200 pqu) , o tempo para incapacitação é muito arrto, cerca de 2 minutos. Além do mais, o Hav é um gás muito rápido a provocar knock-out aquando dos incêndios, enquanto que o co habitualmente actua mais sustentadamente no tempo (Penney, 2000). Quando uma vítima fica incapacitada por AN continuará a inalar CO enquanto estiver viva. Este é o motivo porque se pensa que a maioria das mortes ocorre devido ao co, enquanto que o aN está precisamente por detrás dele.

\section{Reflexão Canclusiva}

Presentemente devemos prestar toda a atenção no terreno às intoxicações quer por $\mathrm{CO}$ quer por HON; durante longuíssimo tempo, houve o entendimento de que as intoxicações estavam directamente relacicnadas como co o que dorigava a uma intervenção terapêtica exclusiva nesse sentido. Desde há cerca de una década, comparticular ênfase nos últimos anos, o Serviço de Saúde deve estar devidamente capacitado para intervir rápida e eficazmente no terreno, commaterial emétodos adequados, não só às intoxicações pelo co, às intoxicações provocadas pelo av, mas também às aditivas ausinérgicas.

\section{Agradecimento}

Os autores agradecedem à Dr.a Tânia Pires a colaboração prestada na composição do texto.

\section{Bibliografia}

Arustine, J. ; Walsh, D. ; (2006) - "Smoke Associated Cyanide Exposure: the importance of ronpt recognition and protocols for prehospital treatment", stroke Elcational (Stpl) August: 15-19.

BandeirR, R. ; RerS, A. M. ; Pance Lẽ̃o, R.; GandR, S. e GandRA, R. (2007) - "O serviço de Saúde nos Bombeiros. A sua Importância, da Univítima à Medicina de Catástrofe", Territorium, 14: 99-108.

Baw, F. (2008) - "Intoxications par les funées d' incenoie". ENC (Elsevier Masson SAS, Paris) , Toxicologie-
Pathologieprofessionelle, 16-539-G-10

DED LoG-Mhrov, R. ; Juncos, Jorge L. (2008) - "Chapter 366. Parkinson's Disease and Other Extrapyramidal Movement Disorders, Secondary Parkinsonism - Drug-Induced Parkinsonism". In Fanct, A. S. ; Brennard, E. ; Kastrer, D. L. ; Hexser, S. L.; Lavo, D. L. ; Javeson, J. L. e Losaño, J. (ed) : Hbrrison's Principles of Intemal Medicine, $17^{\mathrm{a}} \mathrm{ed}$ New York.

ForrIN, J. L. ; Whax, S. ; ArIS, A. -M. ; GrocanII, J. P. ;

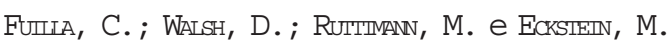
(2006) - "Acute Cyanide Poisoning: a Paris Firefighter recovers from severe smoke inhalation", Stroke Educational (Supl) August: 19-21.

Hoffman, R. ; Newson, L. ; Howland, M. ; LewIN, N. ; Flomenbaum, N. e Goldfrank, L. (2007) Goldfrank's Menual of Toxicologic Energencies, The MbGraw-Hill Companies, Inc. New Youk.

Hirst, C.; Newwpr, J.; Rovnd, Jr. e Janes, A. (2008) "Chemical Bioterrorism". In FAcr, A.; BसRWWD, E.; KASPER, D.; HAUSER, S. ; LoNGO, D. ; Janesan, J. e Losato, J. (ed) : Harrison's Principles of Intemal Medicine, $17^{a}$ ed New York.

IAInN, J. etal., (2009) - "Fire fighting trainer's exposure to carcinogenic agents in smoke diving simulators" . Toxicol. Iett. obi: 10.1016/j.toxlet.2009.06.864.

Ińx, F. (2008) - "Actualités des intoxications aux fumées d'incendie' . Actes Secaurs Santé 2008, Evianp. 14.

Megrabane, P.; Fortin, J. L. e HagahedAF, M. (2008) "Intoxication cyahydrique aigue", In Urgence PratiqueRblications (ed) , Ies intoxications, Priseen darge initiale, IeVigan, pp. 75.

MLreav, D. L. e BRrs, D. J. (2008) - "Poisoning". In Stone, C., Himeries, R. (ed) Current Diagnosis \& Treatment Emergency Medicine, $6^{\text {th }}$ Edition, McGraw Hill, Lange, New York.

Orsav, K. R. (2009) - "Poisoning". In McPefe, S. e PAFIAKIS, M. (ed) 2009 Current Medical Diagnosis \& Treatment, $48^{\text {th }}$ Edition, MbGraw Hilll, Lange, New York, 2009.

OxfordDictionary of Science (2005) $5^{\mathrm{a}}$ ed.

Peney, D. G. (2000) - Carbonn Monoxide Toxicity. CRC Press, Boca Raton, EL.

Paney, D. G. (2009) - "The Toxic Twins: Na Advance Perspective on Cyanide and Carbon Monoxide Poisoning", Smoke Educational (Supl) Merch: 12p.

SAMIRACO, I. (2003 a) - "Intoxicación por gases". In Pnures, M. A. (ed) Anales del Sistema Sanitário de Navarra, 26 (Supl. 1) : 173-180.

SAMIIACO, I. (2003 b) - "Contaminación por agentes químicos". In Panmos, M. A. (ed) Anales del Sistema Sanitário de Navarra, 26 (Supl. 1) : 181-190.

SHEPHERD, G. e VEIEZ, L. I. (2008) - "Role of hydroxocobal amin in aate cyanide poisoning". Am Pharmacother 42 (5) : 661-9. 